\title{
Towards Dementia-friendly Smart Homes
}

\author{
Mohsen Amiribesheli \\ Department of Computing \& Informatics \\ Faculty of Science and Technology \\ Bournemouth University, $U K$ \\ Email: mamiribesheli@bournemouth.ac.uk
}

\author{
Abdelhamid Bouchachia \\ Department of Computing \& Informatics \\ Faculty of Science and Technology \\ Bournemouth University, UK \\ Email: abouchachia@bournemouth.ac.uk
}

\begin{abstract}
Care costs of People with Dementia (PwDs) bear a tremendous burden on healthcare systems around the world. Smart Homes (SHs), as an instance of the ambient assisted living technology, can help reduce these expenses. However, only few of the existing studies in the literature discuss how SHs should be designed by considering the unique requirements of PwDs. Most of the studies view dementia care as a straight application of standard SH technology without accommodating the specific needs of PwDs. A consequence of this approach is the inadequacy and unacceptability of generic SH systems to PwDs as well as other stakeholders in the sector of dementia care. The present research reports on the requirements elicitation, design and evaluation of a dedicated SH prototype for PwDs. In contrast to most of the existing SH systems proposed for PwDs, this work presents a tailored prototype that is based on a user-centred design methodology. The preliminary evaluation by a sample of stakeholders shows the suitability of the proposed methodology and consequently the resulting prototype for reducing care difficulties as well as its potential of deployment in the real-world environment.
\end{abstract}

\section{Introduction}

Dementia is a family of chronic diseases that cause permanent and gradual cognitive decline. Expectations show that one in five people will be older than 65 by 2030 [38]. Also, about $3 \%$ of people aged 65 to $74,19 \%$ of people aged 74 to 84 and nearly half of people more than 84 have dementia [33]. Most of them, however, strongly prefer to stay in their own homes and communities, a phenomenon known as age-in-place. It has been observed that age-inplace can reduce the speed of dementia progress, can improve people's quality of life [7] and can enable them to be surrounded by their families. However, informal care at home can be excessively expensive and in some cases it is not possible at all [39]. Taking these to account, the quality of lives of People with Dementia (PwDs) can be improved by adopting ambient assisted living technologies such as Smart Homes(SHs) [3], [8], [12]. It has been widely accepted that SHs cannot play their potential roles unless they are designed considering precise requirements of their users [10], [32].
SHs for dementia care should be able to address requirements of different users. Commonly, the users are PwDs, informal caregivers (e.g., family members), formal caregivers (e.g., geriatrics) and social caregivers (e.g., staffs of care homes) [11]. Despite the clear importance of this need, the existing literature does not emphasise enough on the problem of requirements elicitation and evaluation [29] when designing SHs for dementia care. Existing studies have mainly focused on certain care scenarios instead of covering the complete analysis of SH requirements for dementia. Mostly, such selected scenarios are designed for particular lab environments and user settings [13].

A viable way to elicit the requirements of PwDs is to employ a User-Centred Design (UCD) approach which involves the users of the system in the specification of their requirements [14]. However, given the peculiarities of dementia disease (e.g., memory restraints and mobility difficulties), it is considerably challenging to involve PwDs in the requirements elicitation steps. Moreover, the collected information from PwDs might not be accurate [24]. On the contrast, informal and formal caregivers are usually wellinformed about PwDs' needs and preferences. Therefore, the requirements elicitation and evaluation should be driven by the caregivers. Nevertheless, observing PwDs' interaction with the system in a long period can be beneficial for evaluating the system in real-world environments [19].

A handful of studies have looked at the specific requirements of PwDs living in SHs. For instance, the authors in [24] analysed the requirements of PwDs using techniques such as interviews and focus groups. The study showed that: a) PwDs cannot learn how to use new devices in their homes. b) For familiarity reasons, if there is a need to deploy a new equipment in the $\mathrm{SH}$, then it is preferable to choose one that is similar to an existing one. c) PwDs prefer to maintain the sense of control over their environment. d) It is recommended that formal caregivers should be the first to evaluate any new technology proposed for PwDs. e) Prompts and reminders should be used to notify PwDs about emergency actions.

In an effort to overcome the challenges of requirements elicitation for PwDs, Stephan et al. [18] applied a participatory design method to create a digital aid tool for safe walking. In another study [37], people with mild to 
moderate dementia as well as caregivers were involved in requirements definition of ambient assisted living technologies. It was found that PwDs need support for dressing, taking medicine, maintaining personal hygiene, preparing food and socialising. Van Hoof et al. [35] investigated the effectiveness of SHs for age-in-place using interviews and observation. In this study, an SH prototype was developed and used to analyse a group of old people. The prototype was equipped with various functionalities such as movement monitoring, fire detection, wandering detection and fall detection. It was found that PwDs appreciate safety and security, especially the fall detection. Mihailidis et al. [20] studied the application of SHs for the purpose of assisting people with moderate dementia in the accomplishment of daily living activities. The authors evaluated an audio-visual system dedicated to the task of hand-washing in a bathroom. The system uses video processing techniques to perceive how the washing activity is done. The system can remind the person or call the caregiver if the person could not follow the washing instructions.

Kaye [15] suggested that monitoring dementia progress via sensor data reveals new information which can be adopted to develop more efficient prevention treatments. To establish the point, the author compared data collected by the sensors and conventional assessment approaches and concluded the sensor data is illustrating meaningful change over time. Morris et al. [21] reviewed health technology advances in three areas of monitoring, compensation, and prevention. Results related to ethnography and feedback suggested that adoption of health technologies will increase if monitoring is woven into preventive and compensatory health applications.

Numerous studies highlight the fact that any trivial mistakes in the design and developing phases of an $\mathrm{SH}$ for dementia care could be costly and could prevent stakeholders from adopting it in real-world settings [2]. Additionally, the lack of prior evaluation could put SH residents' safety at risk [1]. Therefore, a series of precise evaluations should be conducted to ensure the effectiveness and harmlessness of the $\mathrm{SH}$ [36].

The principal objectives of this research are to elicit requirements of PwDs living in $\mathrm{SHs}$ by applying tools based on the UCD methodology (See Sec. 2), to develop a functional prototype based on the elicited requirements (See Sec. 5), and to evaluate the prototype (See Sec. 6) to ensure the positive effect on users' experiences. At the end, experiences gained from the study are discussed in Sec. 7.

\section{Methodology}

Using conventional requirements elicitation approaches like interviews and focus groups might not lead to full coverage of the requirements, because PwDs can rarely express their needs and desires accurately [4] due to their cognitive decline and because different classes of caregivers are involved. To cope with this situation, the present study proposes a development cycle consisting of the following five phases (See Fig. 1):

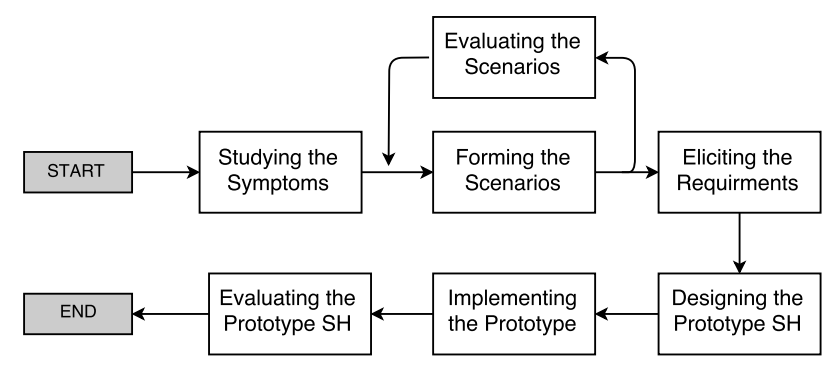

Figure 1. The development stages of the prototype

\subsection{Phase 1: Studying the Symptoms \& Forming the Preliminary Scenarios}

This phase was begun with the study of the existing body of literature on dementia symptoms, during which the symptoms that show PwDs' inability to perform the activities of daily living were collected. Initially, 25 symptoms that needed caregivers' interventions were gathered. This large number of symptoms could make the requirements elicitation process extremely laborious. Also, all the symptoms were not appearing in all the dementia stages [27]. To narrow down the scope, this study concentrated on the dementia symptoms in early (mild) stages. It is widely accepted that in the early stages, PwDs have a greater chance of living independently with the help of well-equipped SHs. Also, monitoring the disease progress at the early stages provides profoundly beneficial information for the treatment process [15]. Ultimately, after excluding the late stage dementia symptoms, a set of 11 symptoms were selected to be used to develop scenarios.

\subsection{Phase 2: Scenario Refining and Initial Evalu- ation}

Making sure that the scenarios were accurate and realistic is extremely critical. To validate the scenarios, the following procedure was adopted. First, four social caregivers and two dementia psychologists were invited to take part at this study. An interview session was held for each interviewee. While reading the scenarios and seeing them being acted in a miniature SH (doll house), they were asked to modify the scenarios details based on their individual experiences. As a result of the interviews, the scenarios were enriched with details, and in a few cases, new scenarios were developed. For instance, "personal grooming" scenario was not on the initial list of scenarios and it was added following a number of suggestions from social caregivers. The final set of scenarios consisted of 11 scenarios (See Table 3.3).

\subsection{Phase 3: Requirements Elicitation}

In this phase, it was attempted to elicit the functional and non-functional requirements of the $\mathrm{SH}$. The functional 
requirements were determined by reformulating the scenarios as use case tables. Each table incorporated the necessary software and hardware components for the $\mathrm{SH}$ to address the needs of each scenario that will serve to design the SH prototype. Also, based on the raised concerns by the interviewees, three non-functional requirements of modularity, distribution and seamless integration were tackled by proposing a group of design considerations.

\subsection{Phase 4: Prototype Implementation}

In this phase, the gathered requirements were embodied in an architecture for the SH. Two key components of hardware and software are needed to implement an SH system. In terms of hardware a group of sensors (e.g., passive infra-red sensors) and a single-board computer were used; while for the software, a system was developed using an existing $\mathrm{SH}$ framework, called OpenHAB, in a way to meet the requirements. The user interfaces were developed from scratch using Java script and PHP programming languages. The interfaces were adapted to mobile phones, tablets and computers.

\subsection{Phase 5: Prototype Evaluation}

A reliable method of evaluating the prototype is to deploy it in real-world environments and observe the interaction of the stakeholders, including the PwDs themselves, for some period of time to assess the impact the quality of their experience [5]. However, before the real-world deployment, it should be guaranteed that the system does not affect the residents' health and safety. This study applied an evaluation method to assess the impact by analysing the collected feedback from the caregivers.

\section{Forming the Scenarios}

As stated in Sec. 1, the study was performed using a User-Centred Design (UCD). In the following, we present $\mathrm{UCD}$ and investigate the process of requirements elicitation and the intervention levels of dementia care.

\subsection{User-Centered Design}

The UCD is an umbrella term that comprises a set of design tools and methods to involve the users of a system to specify the requirements [31]. Therefore, stakeholders should be participating in all stages of the design of an SH including analysis, development and evaluation. In this study, the UCD was acknowledged as the dominant development viewpoint. In the requirements collection phase, the informal and formal caregivers were directly involved in the process by validating the scenarios. In the requirements specification and the prototype development, the caregivers' suggestions were reflected by adopting their requests and concerns. In the evaluation part, the system as a whole was validated by the formal caregivers.
Using the requirements elicitation tools (e.g., scenarios and personas) was not plausible without perceiving the acceptable level of system intervention in the PwDs' daily lives. In the following, we present the dementia care intervention levels and show how they are used for SH systems.

\subsection{Intervention Levels}

An outstanding issue of existing SHs for dementia care is their inability to simulate the caregivers' approaches for intervening in the PwDs' lives. The SHs are often developed to perform the activities of daily living in general which makes them inadequate for PwDs [25].

Caregivers often utilised different levels of intervention depending on particular care circumstances. Ideally, following these levels should support PwDs' independence and guarantee their sense of control. We adopted the following five levels of intervention [28]: inviting awareness, suggesting, prompting, urging, and performing.

- Inviting Awareness: In this level, the system does not take actions on behalf of the PwDs. It merely monitors the activities. Later, the data can be employed to produce reports which are used as the basis for inviting awareness about a situation.

- Suggesting: In this level, the system does not take actions on behalf of PwDs. It only suggests them to choose a particular action. They are free to act on suggestions or take an alternative decision (e.g., a dress to wear). The system does not need to monitor PwD's reactions to the suggestion.

- Prompting: In this level, the system will take actions on behalf of the PwDs only if they ignore its suggestions. The system reminds the PwD to perform an activity in a particular way. Contrary to the "suggesting", the PwDs should not ignore the prompts and if the they do, the system will intervene.

- Urging: In this level, the system performs an activity on behalf of the PwDs and prompts them to act simultaneously. This level is particularly beneficial for tasks that involve both the PwD and the system actions.

- Performing: In this level, the system takes action on behalf the PwDs without any preconditions. Often, this type of intervention happens as a swift reaction to a risky situation.

Usually, caregivers are suggested to have the least interference with the PwDs' lives, and our proposed SH system follows the same approach.

\subsection{Scenarios}

The scenarios are defined as a tangible description of activities that the users engage in when performing specific tasks. Such description is sufficiently detailed so that the design implications can be understood [34]. Scenarios are considered as common UCD tools to collect information about feasible interactions between the users and the system. 
TABLE 1. List OF THE USE CASES

\begin{tabular}{ll} 
Scenario Number & Scenario Name \\
\hline 1 & Exhaustive Repeating Speech \\
2 & Dehydration Risks \\
3 & Communication Difficulties \\
4 & Misplacing Personal Items \\
5 & Losing Personal Items \\
6 & Learning Difficulties \\
7 & Remembering Time and Date Troubles \\
8 & Pacing Risks \\
9 & Night-time Wandering Risks \\
10 & Forgetting Names Frustration \\
11 & Vision Difficulties \\
12 & Confusing Personal Grooming \\
13 & General Monitoring Necessity \\
\hline
\end{tabular}

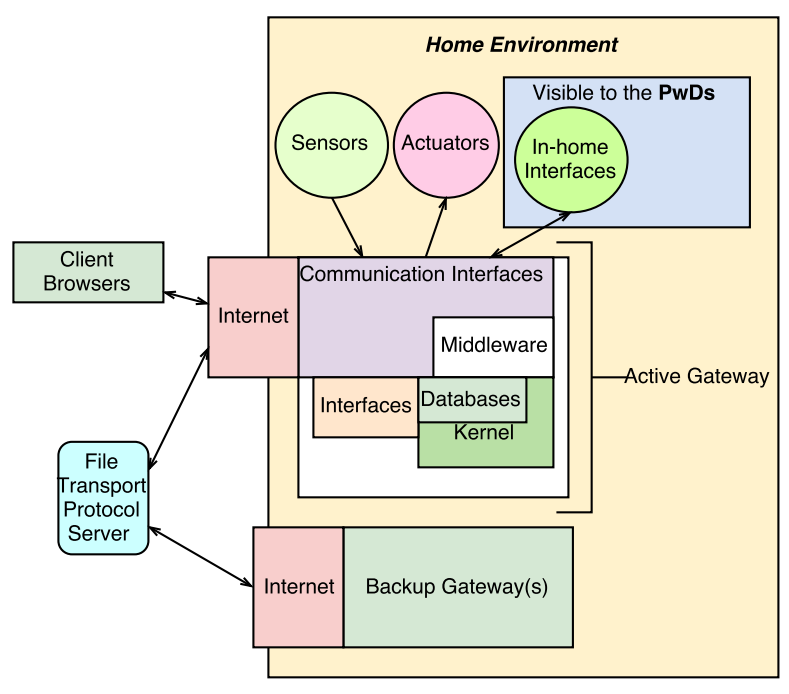

Figure 2. System Architecture

In this study, the process of developing the scenarios consists of the following three steps. First, the most prevalent dementia symptoms are collected from the literature. Then, the scenarios are developed based on the symptoms of each dementia stage using different personas (described in 2.2). Personas enhanced the process of requirements elicitation, especially in the scenario validation phase by making the scenarios simpler to be addressed during the interviews. For instance, instead of referring to an elderly person with the exhaustive repeating speech problem, it was easier to recall the persona "Robert" during the initial interviews and evaluations.

Although, the collected scenarios of this study are based on the most common dementia symptoms, some PwDs experience exceptional cases [11]. Accordingly, the SH should be developed in a flexible manner that allows the stakeholders to define new scenarios at any given time.

\section{Requirements Analysis}

At this stage, the functional requirements were elicited by extracting the scenarios elements and manifesting them in use case tables ${ }^{1}$. Furthermore, the non-functional requirements of modularity, distribution and seamless integration were elicited by analysing the feedback from the interviewees to elaborate a design.

A use case can be described as a list of users' actions and the corresponding reactions of the environment. In this study, a use case is produced for each scenario. The process of analysing the use case leads to the gradual emergence of the necessary software and hardware components of the system. Due to space limitations, only two use cases are presented (see table 2, and table 3 ).

\subsection{Overall Design Considerations}

Non-functional requirements affect the overall operation of the system and have a significant role in its acceptability amongst the users. They are modularity, distribution, and seamless integration as expressed by the interviewees.

4.1.1. Modularity. PwDs share common requirements, but each of them has also specific requirements. The environment changes from one PwD to another and therefore sensing devices change. To cope with the dynamic change and the variability, an open modular design of the system should be considered. A very prominent modular approach that can satisfy these needs is the Service-Oriented Architecture (SOA). SOA empowers the SH with universal services that are platform-independent and reusable. Additionally, the SOA-based SH is extensible, which implies stakeholders can add or remove services at any given time.

4.1.2. Distribution. The SOA-based design patterns were suggested as the appropriate approach to address the modularity requirements of the $\mathrm{SH}$. However, they often utilise a client-server model. It means all processes run on a computing unit (gateway), that puts all the processing load on a single machine. This leads to issues with the SH system, particularly regarding reliability. In the client-server design, the gateway failure causes all the SH services to be unavailable. Distributed design methods prevent these problems by allowing the processing load to be divided into different components. Different distributed design approaches can be used for the SH (e.g., mobile computing, and network file systems, and virtualisation). They enable the SH to be equipped with multiple gateways simultaneously.

4.1.3. Seamless Integration. Putting unfamiliar devices (e.g., sensors and actuators) in a PwD's living environment causes anxiety for them. To prevent this from happening, all the hardware components of an SH system must be seamlessly integrated into the environment. For instance, the communication between PwD and the SH should occur through natural user interfaces or familiar devices such as radio and $\mathrm{TV}$.

1. The use case tables containing brief versions of the scenarios can be found at https://goo.gl/mQBpJO 
TABLE 2. USE CASE 1

\begin{tabular}{|l|l|}
\hline Title & \#1 Repetitive Speech \\
\hline Concept & $\begin{array}{l}\text { The repetitive speech is a prevalent symptom of dementia. Its progress has a direct } \\
\text { correlation with dementia stage. Its tracking helps show the disease progress. }\end{array}$ \\
\hline Rationale of system reactions & $\begin{array}{l}\text { The system invites awareness. It detects the event by analysing the collected data from } \\
\text { sensors (e.g., microphone) [22]. The frequency of repetitive speech outlines the disease } \\
\text { stage; particularly it shows the transition from the Mild Cognitive Impairment (MCI) stage } \\
\text { to the early stages of the Alzheimer's Disease. }\end{array}$ \\
\hline Scenario & $\begin{array}{l}\text { Robert is an elderly person with MCI. He lives in his home with his family. He repeats his } \\
\text { questions and statements inattentively. His GP stated that the progress of dementia increases } \\
\text { the number of speech repetitions. Also, very little can be done by the caregivers to prevent } \\
\text { him from repeating his speech. }\end{array}$ \\
\hline Required components & $\begin{array}{l}\text { A set of microphones to collect the PwD's speech. } \\
\text { A database system to save the logs and recordings. } \\
\text { A processing unit (kernel) to perform the voice recognition using existing web } \\
\text { speech interfaces (e.g., Google web speech API) and to prepare the reports. } \\
\text { An interface to present the reports. }\end{array}$ \\
\hline
\end{tabular}

TABLE 3. USE CASE 2

\begin{tabular}{|l|l|}
\hline Title & \#6 Learning Difficulties \\
\hline Concept & $\begin{array}{l}\text { It is difficult for elderly people to learn how to use previously unseen devices. For PwDs, } \\
\text { the process of learning how to manage the devices (e.g., home appliances) is even more } \\
\text { complicated. Therefore, the system should provide the necessary guides interactively. }\end{array}$ \\
\hline Rationale of system reactions & $\begin{array}{l}\text { The system suggests. It gets activated by analysing the sensors readings. As the PwD begins } \\
\text { to use the newly added device, the system prompts him/her the operation instructions through } \\
\text { the available interfaces (e.g., radio speaker) [20]. }\end{array}$ \\
\hline Scenario & $\begin{array}{l}\text { Virginia is an elderly person with mild dementia living in her home with her family. } \\
\text { She encounters difficulties to cope with new equipments. For instance, she has problems } \\
\text { interacting with the newly added microwave in the kitchen. }\end{array}$ \\
\hline $\begin{array}{l}\text { A set of PIR motion and contact switches (sensors) to recognise the activity of } \\
\text { using the device. }\end{array}$ \\
A database system to save the logs. \\
A processing unit (kernel) to perform the activity recognition and choose the \\
necessary instructions. \\
An interface to be used for playing the instructions.
\end{tabular}

Acknowledging the mentioned requirements and design consideration the system architecture is presented in the Figure 2.

\section{The Prototype}

A prototype was developed based on the architecture introduced in Figure 2. In the following, prototype components and their implementation methods are presented.

\subsection{Hardware Components}

In the following, we introduce the chosen hardware components for the prototype and their configuration in details.

5.1.1. Sensor, Actuators and In-home Interfaces. As shown in the use cases, various sensors, actuators and inhome interfaces can be used to implement the $\mathrm{SH}$ functions. Also, in some instances, a care response can be implemented by utilising different hardware devices. For example, to check the PwD's location, employing both passive infra-red sensors and cameras could be equally beneficial. Nevertheless, in the prototype development only binary sensors were used. The adoption of binary sensors complied more with the PwDs' privacy requirements [9], and it prevented the prototype from being resource-intensive [17].

In the prototype, actuators and in-home interfaces were implemented in a seamless manner (see Sec. 4.1.3) and in a way that they did not require the PwDs to learn complex interaction methods [16]. For instance, instead of employing a speaker for conveying the messages to the PwDs, a digital radio was used.

5.1.2. Gateway. Gateway is a device primarily responsible for receiving the sensor data, running the software components that process them, sending orders to the actuators, and transferring information to the interfaces. Reflecting on the design considerations (See Sec. 4.1), the prototype required a light, cheap, easily replaceable and fault tolerant device as the gateway. Principally, several single board computers could satisfy these needs. The Raspberry Pi B [26] was selected for the prototype implementation. It has a $700 \mathrm{MHz}$ processor, 512 MegaBytes of RAM, and communication 
The following table represents the critically risky situations detected by the system.

\begin{tabular}{|c|c|c|c|}
\hline ID & Date & Message & Current Action \\
\hline$\underline{1830}$ & 09/02/15 2:42 PM & $\begin{array}{l}\text { The PwD used the toilet } 63 \text { times this month which is much lower } \\
\text { than expected (min =90). }\end{array}$ & $\begin{array}{l}\text { Suggesting } \\
\text { Change }\end{array}$ \\
\hline$\underline{1831}$ & 09/02/15 2:42 PM & $\begin{array}{l}\text { The PwD only used the kitchen appliances } 80 \text { times this month which } \\
\text { is much lower than expected ( } \mathrm{min}=160 \text { ). }\end{array}$ & $\begin{array}{l}\text { Suggesting } \\
\text { Change }\end{array}$ \\
\hline$\underline{1833}$ & 09/02/15 2:42 PM & $\begin{array}{l}\text { The PwD repeated a phrase } 40 \text { times which is much more than } \\
\text { expected threshold. Please click on the ID for more details. }\end{array}$ & Urging Change \\
\hline$\underline{2649}$ & $\begin{array}{l}09 / 15 / 1512: 54 \\
\text { PM }\end{array}$ & $\begin{array}{l}\text { The PwD only urinated } 57 \text { times this month which is lower than } \\
\text { expected. He might be in Dehydration risk }\end{array}$ & $\begin{array}{l}\text { Suggesting } \\
\text { Change }\end{array}$ \\
\hline
\end{tabular}

Figure 3. Prototype interface: Critical section of the main report.

ports such USB and General-Purpose Input/Output (GPIO). A non-graphical version of Linux operating system, a web server, a middleware, and a database system were installed on it. As mentioned earlier (see Sec. 4.1.2), distribution is an essential requirement of SHs for dementia care. To address this, the gateway was configured in a way that the settings and collected data were automatically duplicated on another machine utilising file transfer protocol. The backup machine could function as the active gateway at any given time (See Fig. 2).

\subsection{Software Components}

In the following, we explain the software components of the prototype.

5.2.1. Communication. The prototype required a diverse set of communication platforms and protocols to enable inhome and remote communication amongst its components. Remote communication services are in charge of providing the connection between the web server installed on the gateway and the remote interfaces (e.g., mobile devices and computers). Accepting the internet as the primary remote communication platform was the most convenient approach. In this study, the Raspberry Pi wireless networking capabilities were utilised to connect the system to the internet. Since some of the collected data are sensitive, their security is a very common concern [6]. The prototype addressed the security-related concerns by adopting a Linux-based firewall.

There exists a variety of sensor networking protocols for in-home communication because each sensor or actuator might require a proprietary networking protocol. Therefore, it is the middleware role to enable the rest of system to communicate with indoor devices. For the prototype sensors,
GPIO communication protocol was used. Also, the in-home interfaces (e.g., digital radio) utilised the local area wireless networking protocol provided by the gateway.

5.2.2. Middleware. The middleware of an $\mathrm{SH}$ is a software component that is responsible for bridging the physical devices (e.g., sensors) with the rest of the components running on the gateway. Also, it is expected from the $\mathrm{SH}$ middleware to guarantee that the processes of discovering, adding and configuring the new hardware devices to the SH should occur without the need for restarting or reconfiguration of the system (See Sec. 4.1.1). There are open source middleware systems for ambient assisted living that could be adjusted to meet the necessary requirements of the prototype [30], [40]. We opted for this solution instead of developing a new middleware from scratch. The open Home Automation Bus (openHAB) [23] is an open source middleware based on open service gateway initiative, which is one of the most common approaches to implement SOA. It was customised to meet all the requirements of the study. As personalised interfaces were developed, none of open$\mathrm{HAB}$ interfaces were used in the prototype. The openHAB supports a substantial number of communication protocols for sensors and actuators including GPIO, Bluetooth, and Z-wave. It was adjusted in a way that all sensors activities were collected in a database that the system kernel processed them. Also, kernel utilised the Representational State Transfer Application Program Interface (REST API) to control the actuators using the openHAB as its middleware.

5.2.3. The Kernel. The kernel of an $\mathrm{SH}$ is a software component that is responsible for processing the collected data and determining the system reactions to particular situations. The prototype kernel was performing the decisionmaking task by employing a Fuzzy Rule-based (FR) model. 
John's sleep type from 12:00 am to 8:00 am on 20/09/2015

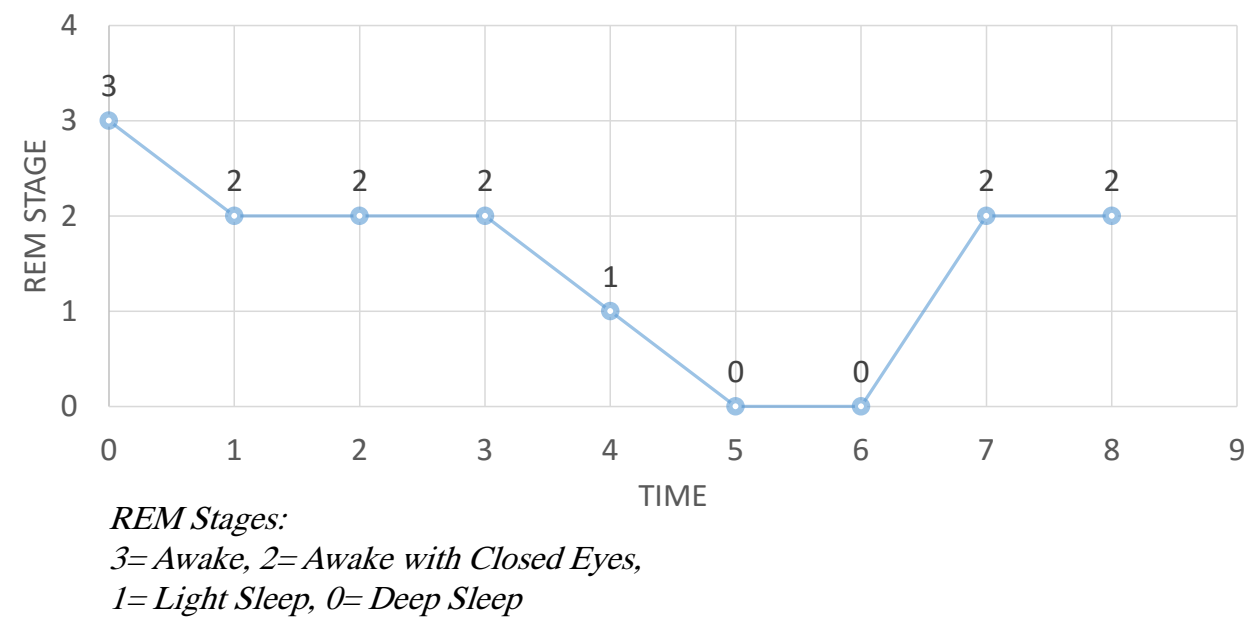

Figure 4. Prototype interface: Sleep type per hour.

It was developed with Python programming language. The FR model was utilised to enable the caregivers to create rules using linguistic variables (e.g., too many times, high, and low). The model requires membership functions to map linguistic variables with ranges of collected data in the datasets. To do that, a group of formal caregivers was asked (five geriatrics) regarding their interpretations of healthcare sign reading of different common categories of PwDs (e.g., a male PwD with high blood pressure, a female PwD with Parkinson's disease). As an instance, for a male PwD with diabetes type 2, the ranges for low, normal, and high blood glucose is not the same with a male PwD without diabetes. Next, a profile was associated to each of the PwDs' categories. Ultimately, the FR model and the profiles allowed the caregivers to define rules with linguistic variables employing the interfaces. For instance, the caregiver could set a rule such as "if the PwD took his diabetes pills and the blood glucose is very high or very low then it is a risky situation". It is worthy to mention that the system is designed in a way that formal caregivers should personalise or confirm the health thresholds for each PwD.

5.2.4. The Interfaces. Acknowledging the variety of stakeholders and their requirements, it was essential to develop the prototype as a cross-platform application. To develop the interfaces, web development programming languages, PHP and Javascript were used. The quality of interaction with the interfaces dramatically affects the stakeholders' experience. Therefore, the interfaces were fully developed for three platforms: computers, tablets, and mobile phones. Following the authentication of the user, each caregiver will be presented with a list of PwDs who they care for. After selecting the PwD, the facts data about the activity and the health of the selected PwD are displayed. Also, on the same page, some health alerts are presented. Figure 3 ) shows the screen that allows the caregivers to adjust the intervention level for an alert. In case the type of action is not adjusted by the carer, the system performs the default intervention. For instance, if the geriatric set the threshold for the daily urination times to five and the data illustrated less than that, then the system will assume that the PwD is not drinking enough water. Hence, it will play a prerecorded audio reminder to the PwD via a digital radio in the bedroom. Furthermore, an email alert will be sent to the caregivers for information. By clicking on each alert, the relevant data is visualised. For instance, the figure 4 presents the PwDs' sleeping hours and types collected $(0=$ deep sleep, $4=$ awake $)$ by a mobile phone device located on the bed.

\section{Evaluation}

Five geriatric specialists participated in two 45-minutelong evaluation sessions in which they were presented with a prototype SH (See Figure 5). The prototype was equipped with three sets of sensor devices including two groups of binary sensors (Passive Infra-Reds (PIRs) and Contact Switch Sensors (CSSs)) and an e-health monitoring kit that included sensors for vital signs monitoring such as SPO2, airflow, body temperature, and blood pressure. Binary sensors and the e-health sensors were used to populate the databases through the process of acting the scenarios in the doll house. The participants could interact with the prototype using a tablet, and a PC.

The key objectives of the evaluation were to answer the following questions based on the participants feedbacks:

- Does the system have the adequate monitoring capability to be installed in a real-world setting?

- Can the system reduce the care difficulty?

- Does the system consider PwDs' health and safety? 


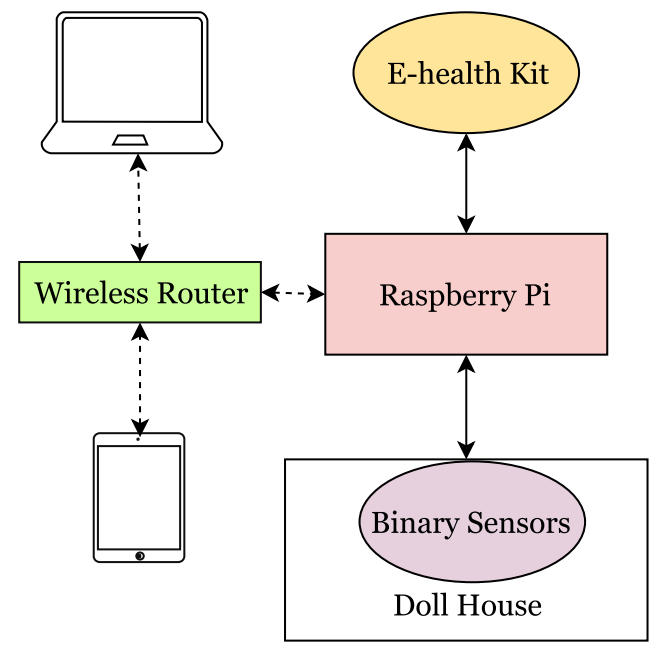

Figure 5. The physical setup of the evaluation prototype.

- Can the system improve the quality of dementia care?

In the following, we provide the details of the evaluation process.

\subsection{Evaluation Approach}

Two evaluation sessions were held for each caregiver. We asked them to answer the questions before and after being introduced to the prototype. In the first session, we examined the importance, the difficulty, and effect of care actions on PwDs assuming that the caregivers performed them. In the second session, we investigated the difficulty and effect of the care actions on the PwDs assuming that the system performed them.

Three measures were utilised in the questionnaires. The "importance" and the "difficulty" were adopted from the study [36], while "effect" was introduced in this study.

6.1.1. Importance. The measure is applied to estimate the overall importance of a care response. It examines the importance from monitoring PwDs and compensating their limited capabilities. Moreover, it enables the participants to judge the relevance of questions. The scale of this measure ranges from 0 to 5, 0 indicating that a care response does not have any importance at all, and 5 stating that it is significantly important.

6.1.2. Difficulty. The measure is applied to examine the difficulty of performing a care action with or without using the SH system. Naturally, it is anticipated to observe a significant drop in the difficulty level, after the $\mathrm{SH}$ is introduced to the participants. The scale ranges from 1 to 5,1 meaning that the care response is quite easy to be performed and 5 indicating that it is extremely difficult to perform it.
6.1.3. Effect. The measure is applied to investigate the effect of performing a care action of the caregivers or the $\mathrm{SH}$ on the wellbeing of PwDs. It is expected for the care responses with higher importance to have a greater effect on PwDs. Using technology may result in a novel type of care that did not exist before. Therefore, its effects on the PwDs might change. The scale range from 1 to 5,1 meaning that the care response does not have any effect on the wellbeing of PwDs and 5 suggesting that it has a major effect on them.

\subsection{Results}

48 questions were asked from five participants in the first evaluation round. The questions included three sets of 16 items regarding the importance, the difficulty, and the effect of the care responses. In the second evaluation round, after introducing the $\mathrm{SH}$ to the participants, 36 questions were related to the difficulty and the effect of care responses. Therefore, each participant responded to 84 questions. As an instance, Figure 6 illustrates the study questions regarding the dehydration risk for PwDs. Based on the answers, a difficulty score $D_{(\text {score })}$ and an effectiveness score $E_{(\text {score })}$ were calculated using the following expressions:

$$
\begin{gathered}
D_{(\text {score })}=\text { "importance" * "difficulty" } \\
E_{(\text {score })}=\text { "importance" * "effect" }
\end{gathered}
$$

For instance, the second participant, a geriatric specialist with 20 years of experience, supposed the results of monitoring repetitive speech symptoms provided critical diagnosis information $(i=5)$. He thought it was troublesome for the caregivers to record the change in the frequency of PwD's repetitive speech $\left(d_{1}=4\right)$. Considering the possible imperfections of the manually collected data, reviewing them could have little effect on his diagnosis and consequently, on the quality of dementia care $\left(e_{1}=3\right)$. After being presented with the SH interfaces, he thought the difficulty of collecting the fluctuations dropped drastically $\left(d_{2}=1\right)$. Moreover, he would trust the sensor collected data more than manually collected data and the visualisation made the changes more explicit. Hence, it could have a more significant impact on his diagnosis and the quality of care $\left(e_{2}=4\right)$.

$$
\begin{gathered}
D_{(\text {score })(\text { Round } 1)}=5 * 4=20 \\
E_{(\text {score })(\text { Round } 1)}=5 * 3=15 \\
D_{(\text {score })(\text { Round } 2)}=5 * 1=5 \\
E_{(\text {score })(\text { Round } 2)}=5 * 4=20
\end{gathered}
$$

Figure 7 and Figure 8 present the average D and E scores for each participant. The average of difficulty score was 15.1 in the first round of evaluation. It slashed drastically to 5.6 in the second round. Moreover, the average of effectiveness score was 16.2 which increased to 16.73 in the second round of evaluation. 


\begin{tabular}{|r|l|}
\hline \multicolumn{2}{|c|}{ \#1. Dehydration Risk Questions } \\
\hline Round 1 & Before being introduced to the prototype: \\
\hline 1 & $\begin{array}{l}\text { How important is it to remind the PwD to drink enough } \\
\text { water? }\end{array}$ \\
\hline 2 & $\begin{array}{l}\text { How difficult is it for the caregivers to remind him/her to } \\
\text { drink enough water? }\end{array}$ \\
\hline 3 & $\begin{array}{l}\text { What kind of effects does the reminding process have on } \\
\text { the quality of PwD's care? }\end{array}$ \\
\hline Round 2 & After being introduced to the prototype: \\
\hline 1 & $\begin{array}{l}\text { Considering the SH functions, how difficult is it for the } \\
\text { system to remind the PwD to drink enough water? }\end{array}$ \\
\hline 2 & $\begin{array}{l}\text { What kind of effects does the reminding process have on } \\
\text { the quality of PwD's care? }\end{array}$ \\
\hline
\end{tabular}

Figure 6. Study questions regarding the dehydration risk

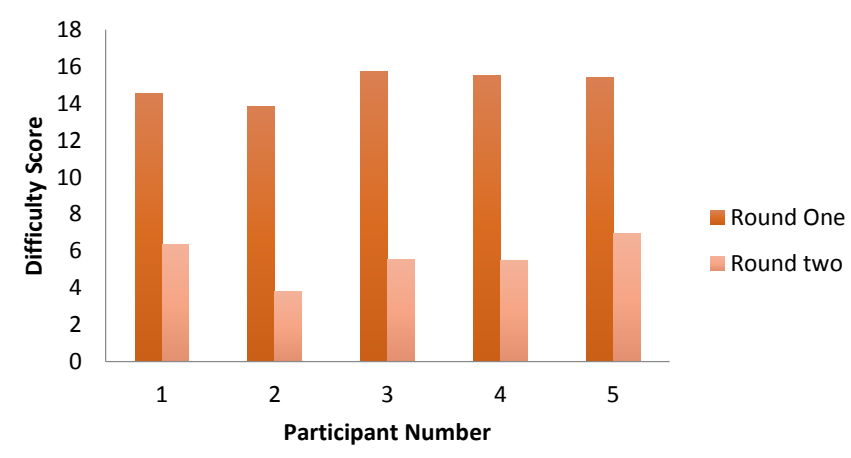

Figure 7. Average D scores for each participant in the two evaluation sessions

\section{Discussion}

Initiating the requirements elicitation by directly interviewing the stakeholders was our preferred approach at the beginning of the study. However, from early stages in the process, two fundamental flaws were discovered with this approach. First, PwDs might not be able to express all of their needs in detail. Second, caregivers often have not dealt with all of the dementia symptoms, and considering the vast number of symptoms, they could easily forget to mention some of them during interview sessions. To overcome these shortcomings, a novel approach using UCD tools and creativity triggers was undertaken. All the symptoms were gathered from the literature, and they were transformed into the scenarios. They were acted for the caregivers using a doll house. Consequently, a better participation and richer feedback from the caregivers were obtained. Eventually, we had a comprehensive collection of care scenarios that were accepted by all the participants of the design and prototype evaluation sessions.

The standard requirements engineering tools (e.g., use cases) were applied to obtain the SH components. Also, information collected from the literature and the interviews with caregivers revealed that the non-functional requirements, such as modularity, played a significant role in the acceptability of the SH by its stakeholders. These considerations could not be reflected simply by introducing a group of components, but they affected the whole system.

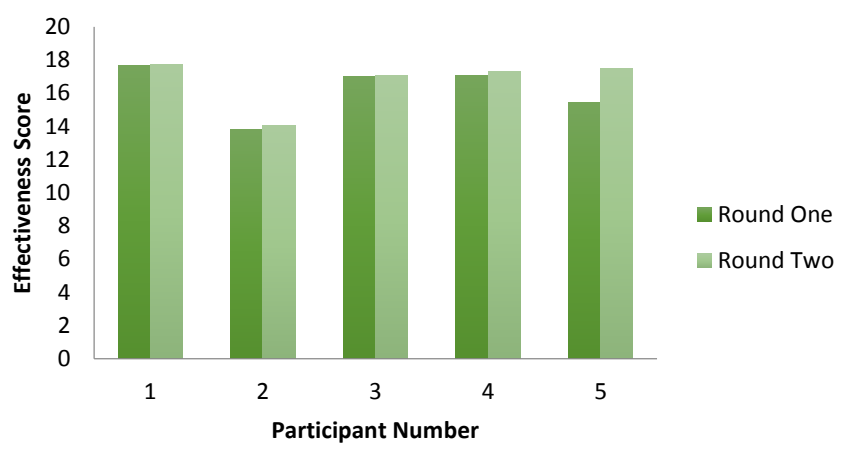

Figure 8. Average $\mathrm{E}$ scores for each participant in the two evaluation sessions

The modularity, distribution, and seamless integration of devices were considered as non-functional requirements to be considered during the system design. We believe that ignoring any of these considerations will negatively affect its acceptability.

Considering the results shown in Sec. 6.2, it is fair to assume that the SH can reduce the difficulties of dementia care dramatically. Hence, it increases the caregivers' peace of mind and allows them to spend more time on tasks that improve the wellbeing of PwDs. Furthermore, the results suggest utilising the SH technology does not cause any effect on the quality of the care responses. However, we need to consider that all the participants were formal caregivers. Their view of care is more pragmatic compared to other stakeholders and they are not directly involved with the PwDs' everyday life. Besides, it is rather difficult to predict the long-term impact of using the $\mathrm{SH}$ technologies without observing it real-world settings. During the preliminary evaluation, all the participants unanimously agreed that the prototype is safe and has the required capabilities to be installed in a real-world setting.

This study was successful in meeting its defined objectives. However, as some challenges will only be faced when the system is deployed, in the next step, the SH system should be installed in a real-world environment. For instance, the prototype flawless success in detecting the macro-contexts (e.g., the risk of night-time wandering) was based on the fact the micro-contexts (e.g., walking, and sleeping) were recognised from the clean simulated data. However, in a real setting the micro-context recognition is a much more challenging task.

\section{References}

[1] Giovanni Acampora, Diane J Cook, Parisa Rashidi, and Athanasios V Vasilakos. A survey on ambient intelligence in healthcare. Proceedings of the IEEE, 101(12):2470-2494, 2013.

[2] Mohsen Amiribesheli, Asma Benmansour, and Abdelhamid Bouchachia. A review of smart homes in healthcare. Journal of Ambient Intelligence and Humanized Computing, 6(4):495-517, 2015.

[3] Mohsen Amiribesheli and Abdelhamid Bouchachia. Smart homes design for people with dementia. In Intelligent Environments (IE), 2015 International Conference on, pages 156-159. IEEE, 2015. 
[4] Arlene Astell, Norman Alm, Gary Gowans, Maggie Ellis, Richard Dye, and Phillip Vaughan. Involving older people with dementia and their carers in designing computer based support systems: some methodological considerations. Universal Access in the Information Society, 8(1):49-58, 2009.

[5] Stefan Carmien, Melissa Dawe, Gerhard Fischer, Andrew Gorman, Anja Kintsch, and James F Sullivan Jr. Socio-technical environments supporting people with cognitive disabilities using public transportation. ACM Transactions on Computer-Human Interaction (TOCHI), 12(2):233-262, 2005.

[6] Haowen Chan and Adrian Perrig. Security and privacy in sensor networks. Computer, 36(10):103-105, 2003.

[7] Malcolm P Cutchin. The process of mediated aging-in-place: A theoretically and empirically based model. Social Science \& Medicine, 57(6):1077-1090, 2003.

[8] George Demiris, Brian K Hensel, et al. Technologies for an aging society: a systematic review of smart home applications. Yearb Med Inform, 3:33-40, 2008.

[9] George Demiris, Marilyn J Rantz, Myra A Aud, Karen D Marek, Harry W Tyrer, Marjorie Skubic, and Ali A Hussam. Older adults' attitudes towards and perceptions of'smart home'technologies: a pilot study. Informatics for Health and Social Care, 29(2):87-94, 2004.

[10] William Green, Diane Gyi, Roy Kalawsky, and David Atkins. Capturing user requirements for an integrated home environment. In Proceedings of the Third Nordic Conference on Human-computer Interaction, NordiCHI '04, pages 255-258, New York, NY, USA, 2004. ACM.

[11] Kirstie Hawkey, Kori M Inkpen, Kenneth Rockwood, Michael McAllister, and Jacob Slonim. Requirements gathering with alzheimer's patients and caregivers. In Proceedings of the 7th international ACM SIGACCESS conference on Computers and accessibility, pages 142149. ACM, 2005.

[12] Sumi Helal, William Mann, Jeffrey King, Youssef Kaddoura, Erwin Jansen, et al. The gator tech smart house: A programmable pervasive space. Computer, 38(3):50-60, 2005.

[13] SS Intille, P Kaushik, and R Rockinson. Deploying context-aware health technology at home: Human-centric challenges. HumanCentric Interfaces for Ambient Intelligence, 22, 2009.

[14] Bonnie E John and Dario D Salvucci. Multipurpose prototypes for assessing user interfaces in pervasive computing systems. Pervasive Computing, IEEE, 4(4):27-34, 2005.

[15] Jeffrey Kaye. Home-based technologies: a new paradigm for conducting dementia prevention trials. Alzheimer's \& Dementia, 4(1):S60S66, 2008.

[16] Tiiu Koskela and Kaisa Väänänen-Vainio-Mattila. Evolution towards smart home environments: empirical evaluation of three user interfaces. Personal and Ubiquitous Computing, 8(3-4):234-240, 2004.

[17] Joshua Lifton, Mark Feldmeier, Yasuhiro Ono, Cameron Lewis, Joseph Paradiso, et al. A platform for ubiquitous sensor deployment in occupational and domestic environments. In Information Processing in Sensor Networks, 2007. IPSN 2007. 6th International Symposium on, pages 119-127. IEEE, 2007.

[18] Stephen Lindsay, Katie Brittain, Daniel Jackson, Cassim Ladha, Karim Ladha, and Patrick Olivier. Empathy, participatory design and people with dementia. In Proceedings of the SIGCHI Conference on Human Factors in Computing Systems, CHI '12, pages 521-530, New York, NY, USA, 2012. ACM.

[19] Ahmad Lotfi, Caroline Langensiepen, Sawsan M Mahmoud, and M Javad Akhlaghinia. Smart homes for the elderly dementia sufferers: identification and prediction of abnormal behaviour. Journal of Ambient Intelligence and Humanized Computing, 3(3):205-218, 2012.

[20] Alex Mihailidis, Jennifer N Boger, Tammy Craig, and Jesse Hoey. The coach prompting system to assist older adults with dementia through handwashing: An efficacy study. BMC geriatrics, 8(1):28, 2008 .
[21] Margaret Morris, Stephen S Intille, and Jennifer S Beaudin. Embedded assessment: overcoming barriers to early detection with pervasive computing. In Pervasive Computing, pages 333-346. Springer, 2005.

[22] Vanda Nemes, Dragana Nikolic, Anna Barney, and Peter Garrard. A feasibility study of speech recording using a contact microphone in patients with possible or probable alzheimer's disease to detect and quantify repetitions in a natural setting. Alzheimer's \& Dementia: The Journal of the Alzheimer's Association, 8(4):P490-P491, 2012.

[23] OpenHab. A vendor and technology agnostic open source automation software for your home., Sep 2015

[24] Roger Orpwood, C Gibbs, Timothy Adlam, Richard Faulkner, and D Meegahawatte. The design of smart homes for people with dementiauser-interface aspects. Universal Access in the information society, 4(2):156-164, 2005.

[25] François Portet, Michel Vacher, Caroline Golanski, Camille Roux, and Brigitte Meillon. Design and evaluation of a smart home voice interface for the elderly: acceptability and objection aspects. Personal and Ubiquitous Computing, 17(1):127-144, 2013.

[26] Raspberrypi. Raspberry pi, Sep 2015.

[27] Barry Reisberg, Steven H Ferris, Mony J de Leon, and Thomas Crook. The global deterioration scale for assessment of primary degenerative dementia. The American journal of psychiatry, 1982.

[28] Martin EP Seligman. Helplessness: On depression, development, and death. WH Freeman/Times Books/Henry Holt \& Co, 1975.

[29] Kristen Shinohara and Jacob O Wobbrock. In the shadow of misperception: assistive technology use and social interactions. In Proceedings of the SIGCHI Conference on Human Factors in Computing Systems, pages 705-714. ACM, 2011.

[30] Mohammad-Reza Tazari, Francesco Furfari, Álvaro Fides-Valero, Sten Hanke, Oliver Höftberger, Dionisis Kehagias, Miran Mosmondor, Reiner Wichert, and Peter Wolf. The universaal reference model for aal. Handbook of Ambient Assisted Living, 11:610-625, 2012.

[31] Ergonomics Technical Committee ISO/TC 159. Human-centred design for interactive systems iso 9241-210, 2010.

[32] Mary Frances Theofanos and Janice Ginny Redish. Bridging the gap: between accessibility and usability. interactions, 10(6):36-51, 2003.

[33] Darcy Ann Umphred, Rolando T Lazaro, Margaret Roller, and Gordon Burton. Neurological rehabilitation. Elsevier Health Sciences, 2013.

[34] Joy Van Helvert and Chris Fowler. Scenario-based user needs analysis. chimera working paper, 2, 2003.

[35] TLM Van Kasteren, Gwenn Englebienne, and Ben JA Kröse. Human activity recognition from wireless sensor network data: Benchmark and software. In Activity recognition in pervasive intelligent environments, pages 165-186. Springer, 2011.

[36] Roelof Wessels, Jan Persson, O Lorentsen, Renzo Andrich, Massimo Ferrario, Wija Oortwijn, Taeke VanBeekum, Hakan Brodin, and Luc de Witte. Ippa: Individually prioritised problem assessment. Technology and Disability, 14(3):141-145, 2002.

[37] Joseph P Wherton and Andrew F Monk. Technological opportunities for supporting people with dementia who are living at home. International Journal of Human-Computer Studies, 66(8):571-586, 2008.

[38] Sarah Wild, Gojka Roglic, Anders Green, Richard Sicree, and Hilary King. Global prevalence of diabetes estimates for the year 2000 and projections for 2030. Diabetes care, 27(5):1047-1053, 2004.

[39] Anders Wimo, Bengt Winblad, and Linus Jönsson. The worldwide societal costs of dementia: Estimates for 2009. Alzheimer's \& Dementia, 6(2):98-103, 2010.

[40] Peter Wolf, Andreas Schmidt, Javier Parada Otte, Michael Klein, Sebastian Rollwage, Birgitta König-Ries, Torsten Dettborn, and Aygul Gabdulkhakova. openaal-the open source middleware for ambientassisted living (aal). In AALIANCE conference, Malaga, Spain, pages

$1-5,2010$. 